\title{
Nonviral delivery of synthetic siRNAs in vivo
}

\author{
Saghir Akhtar ${ }^{1,2}$ and Ibrahim F. Benter 2 \\ ${ }^{1}$ SA Pharma, Sutton Coldfield, West Midlands, United Kingdom. \\ 2Department of Pharmacology and Toxicology, Faculty of Medicine, Kuwait University Health Sciences Center, Safat, Kuwait.
}

\begin{abstract}
Sequence-specific gene silencing using small interfering RNA (siRNA) is a Nobel prize-winning technology that is now being evaluated in clinical trials as a potentially novel therapeutic strategy. This article provides an overview of the major pharmaceutical challenges facing siRNA therapeutics, focusing on the delivery strategies for synthetic siRNA duplexes in vivo, as this remains one of the most important issues to be resolved. This article also highlights the importance of understanding the genocompatibility/toxicogenomics of siRNA delivery reagents in terms of their impact on gene-silencing activity and specificity. Collectively, this information is essential for the selection of optimally acting siRNA delivery system combinations for the many proposed applications of RNA interference.
\end{abstract}

\section{Introduction}

Targeted posttranscriptional gene silencing by RNA interference (RNAi) represents a promising new approach for the inhibition of gene expression in cell culture and in vivo. Since its discovery by Fire et al. in 1998 (1), this Nobel prize-winning technology has rapidly emerged as a promising new strategy for drug target validation and the study of functional genomics, and it is currently being evaluated in clinical trials as a potential therapy for diseases of a genetic etiology (2-11). Similar to antisense nucleic acid-based strategies for gene silencing $(12,13)$, RNAi is a process by which a specific mRNA is targeted for degradation as a means of inhibiting the synthesis of the encoded protein.

The RNAi response is triggered by the presence of double-stranded RNA (dsRNA; over $100 \mathrm{nt}$ ) in cells. The dsRNA is degraded into short double-stranded fragments (approximately 21-23 nt long) known as small interfering RNA (siRNA) by an RNAse III-type enzyme, Dicer. The siRNA generated enters the RNA-induced silencing complex (RISC), which becomes activated upon guide (antisense) strand selection (14). Guide strand selection is based on the relative thermodynamic stabilities of the two duplex ends and it is the least stable $5^{\prime}$ end of the duplex that is recognized and asymmetrically unwound by the Piwi-Argonaute-Zwille (PAZ) domain of argonaute 2 , a multifunctional protein within the RISC. The incorporated strand acts as a guide for the activated RISC complex to selectively degrade the complementary mRNA. The argonaute 2 protein this time is responsible for mRNA cleavage via its PIWI domain, which adopts an RNase $\mathrm{H}-$ like structure $(10,15)$. Unfortunately, introduction of long (>30 nts) dsRNA into mammalian cells leads to the initiation of the antiviral IFN response and global protein expression shutdown (4-10). Thus for therapeutic applications RNAi can be effected through delivery of shorter siRNAs that are produced synthetically ex vivo and can lead to highly specific and potent gene silencing in vivo.

Due to its large molecular weight $(\sim 13 \mathrm{kDa})$ and polyanionic nature ( $\sim 40$ negative phosphate charges), naked siRNA does not freely cross the cell membrane, and thus delivery systems are required to facilitate its access to its intracellular sites of action. It is now widely recognized that efficient intracellular siRNA delivery to target sites in the body

Nonstandard abbreviations used: AMD, age-related macular degeneration; BBB, blood-brain barrier; CPP, cell-penetrating peptide; PEG, polyethylene glycol; PEI, polyethyleneimine; RES, reticuloendothelial system; RNAi, RNA interference; siRNA, small interfering RNA; SNALP, solid nucleic acid lipid particle.

Conflict of interest: The authors have declared that no conflict of interest exists. Citation for this article: J. Clin. Invest. 117:3623-3632 (2007). doi:10.1172/JCI33494. following systemic administration is the most important hurdle for widespread use of RNAi in the clinic (4-7, 16-19). Thus the main focus of this Review is to summarize the current understanding of the cellular uptake and delivery of exogenous, chemically synthesized siRNA for in vivo applications (for a review of the related intracellular gene-silencing effectors such as microRNAs and siRNA precursors that are introduced into cells as plasmids or expressed via viral delivery vectors, the reader is referred to other texts [refs. 20-23]).

\section{Pharmaceutical challenges to effective gene silencing in vivo}

There are several challenges that need to be overcome if exogenous siRNA is to be widely used as a therapeutic agent (Figure 1).

Effective design. The siRNA has to be effectively designed so as to target hybridization-accessible sites within the target mRNA and to avoid unintended (off-target) effects $(18,24-33)$. Not all complementary sequences to a targeted mRNA are effective, and thus a combination of computer algorithms and empirical testing have been employed to define potent siRNAs (16). Off-target effects can arise if partial sequence homology, especially within the 3'UTR, exists with mRNAs other than the intended target mRNA and/ or through stimulation of the innate immune response. siRNA duplexes housing certain GU-rich sequences (e.g. UGUGU) can induce IFN- $\alpha$, IL-6, and TNF- $\alpha$ via interaction with TLRs (33). Any effective siRNA design strategy must account for such off-target effects, but since not all immune stimulatory RNA motifs have been identified as yet, empirical testing in the target system is still necessary for identifying specific and potent siRNAs.

Biological stability. Unmodified, naked siRNAs are relatively unstable in blood and serum, as they are rapidly degraded by endo- and exonucleases, meaning that they have short half-lives in vivo. Typically, chemical modifications can be introduced into the RNA duplex structure so as to enhance biological stability without adversely affecting gene-silencing activity. Alternatively, they can be formulated with a delivery system that not only enhances cell uptake but also affords biological stability (see Formulation with a delivery system). Several chemical modifications to the backbone, base, or sugar of the RNA have been employed to enhance siRNA stability and activity (2). In this manner, siRNA degradation in serum and/or cells can be delayed from minutes to hours, and together with an appropriate delivery system, gene-silencing activity in vivo can be sustained for several days (2). Judicious and selective chemical modifications in the guide strand have added benefits, in that they can also reduce most off-target effects (34) and alter thermal stability within the various 


\section{i Effective siRNA design and synthesis \\ Aim to avoid immune stimulation and off-target effects \\ Target RNA should be accessible to siRNA

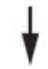 \\ ii Improve biological stability of the siRNA \\ Use chemical modifications and/or a delivery system

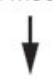

iii Formulate with delivery system

Delivery system must be biocompatible and genocompatible with target

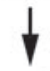

iv Select formulation with most favorable pharmacokinetics and biodistribution

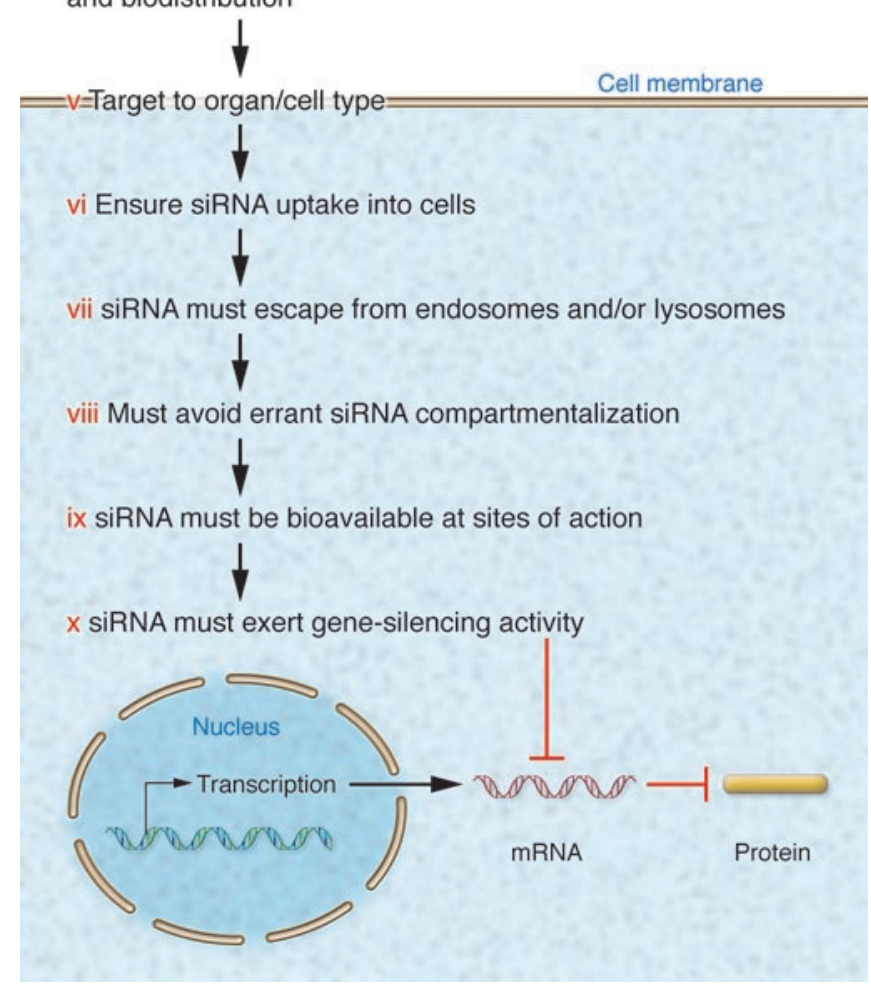

critical sequence regions of the siRNA duplex for improved potency of siRNA (35-37). However, due to the structurally sensitive nature of siRNA duplexes, chemical modifications have to be screened for any loss of potency and enhanced toxicity - e.g., extensive phosphorothioate modifications are known to be toxic $(12,13)$.

Formulation with a delivery system. Considered by many as the most important remaining hurdle to the widespread therapeutic use of RNAi, selection and formulation of siRNA with an appropriate biocompatible and possibly "genocompatible" delivery system is necessary for improving biological stability, targeted cell uptake, and the pharmacokinetics of siRNA (see below for details). Cationic delivery systems can enhance immune stimulation of siRNA (33), and more recently, gene expression array analyses have highlighted the ability of delivery systems to induce a "gene signature" of their own that could increase the off-target effects of siRNA $(18,24,38,39)$. Inappropriate selection of a delivery vector can thereby reduce gene-silencing activity and even enhance off-target effects (18). Delivery systems can also alter the pharmacokinetics of siRNA by altering their molecular and physical size so as

\section{Figure 1}

The pharmaceutical challenges to effective siRNA delivery and activity in vivo. The challenges for effective siRNA delivery and activity in vivo range from the effective empirical or in silico design and selection of an effective siRNA sequence to its chemical modification or formulation with delivery vectors to improve biological stability and pharmacokinetics (steps i-iv). Optimally designed and formulated siRNA should then efficiently enter target cells and escape endosomal and other intracellular compartments to become highly bioavailable inside the cells so as to exert sequence-specific gene-silencing activity with no or minimal effects on nontargeted genes or inadvertent stimulation of the immune system (steps $\mathrm{v}-\mathrm{x}$ ) (see text for detailed explanation).

to reduce excretion via the kidneys and thereby prolong in vivo half-life. Cell targeting systems are available whereby targeting to specific cell surface receptors in specialized tissues is a realistic possibility. Once inside the cell, siRNA has to escape compartmentalization into cell organelles such as endosomes and lysosomes, be intracellularly bioavailable, and interact with its intended mRNA targets in the cytosol to effect highly potent and sequence-specific gene-silencing activity (Figure 1).

\section{Cellular uptake and delivery of siRNA: lessons from in vitro studies}

It is widely thought that cellular uptake of siRNA occurs via nonreceptor-mediated endocytosis, most likely in a manner similar to that observed for other gene-silencing molecules such as oligonucleotides and ribozymes $(12,13,40,41)$. Thus delivery systems are typically used to increase cellular accumulation of siRNA molecules and to facilitate release from endosomes to the cytosol. It should be emphasized that much of the mechanistic and delivery system development studies have been performed in vitro. Although not the focus of this Review (see instead refs. 16-18), important lessons can be learned from these studies that might be useful when progressing to in vivo siRNA delivery. For instance, in vitro studies showed that transfection conditions have to be optimized for each cell model and that efficiency of uptake or genesilencing activity is dependent on variables such as those relating to the duplex siRNA (e.g., chemistry, length, and charge), to the delivery system (e.g., molecular weight, physical size, chemistry, charge, architecture, and shape), to the specific cell culture system (e.g., cell type and growth conditions, including the presence of serum), and to the nature of the target gene/gene product (e.g., its relative abundance and half-life - low abundant targets with short half-lives are thought to be more amenable to silencing). It is worth noting that to achieve greater than $90 \%$ inhibition of the target gene product, the siRNA has to remain effective inside the cell for more than 3 times the half-life of the protein being targeted for silencing. This can require multiple dosing, especially in rapidly dividing cells (42). It is becoming evident that similar optimizations of siRNA delivery for in vivo targets and experimental models need to be performed (16-19).

For in vivo siRNA applications, it was initially thought that naked siRNA was biologically stable enough to be effective without requiring a delivery system (Table 1 ), but more recent studies have highlighted both the need for chemical modification to enhance siRNA stability in the biological milieu and for specific delivery systems that can contribute to improved biological stability but that mainly enhance delivery to target cells and/or organs $(2-8,16-19)$. 
Table 1

Selected examples of delivery systems used for delivering siRNA in vivo

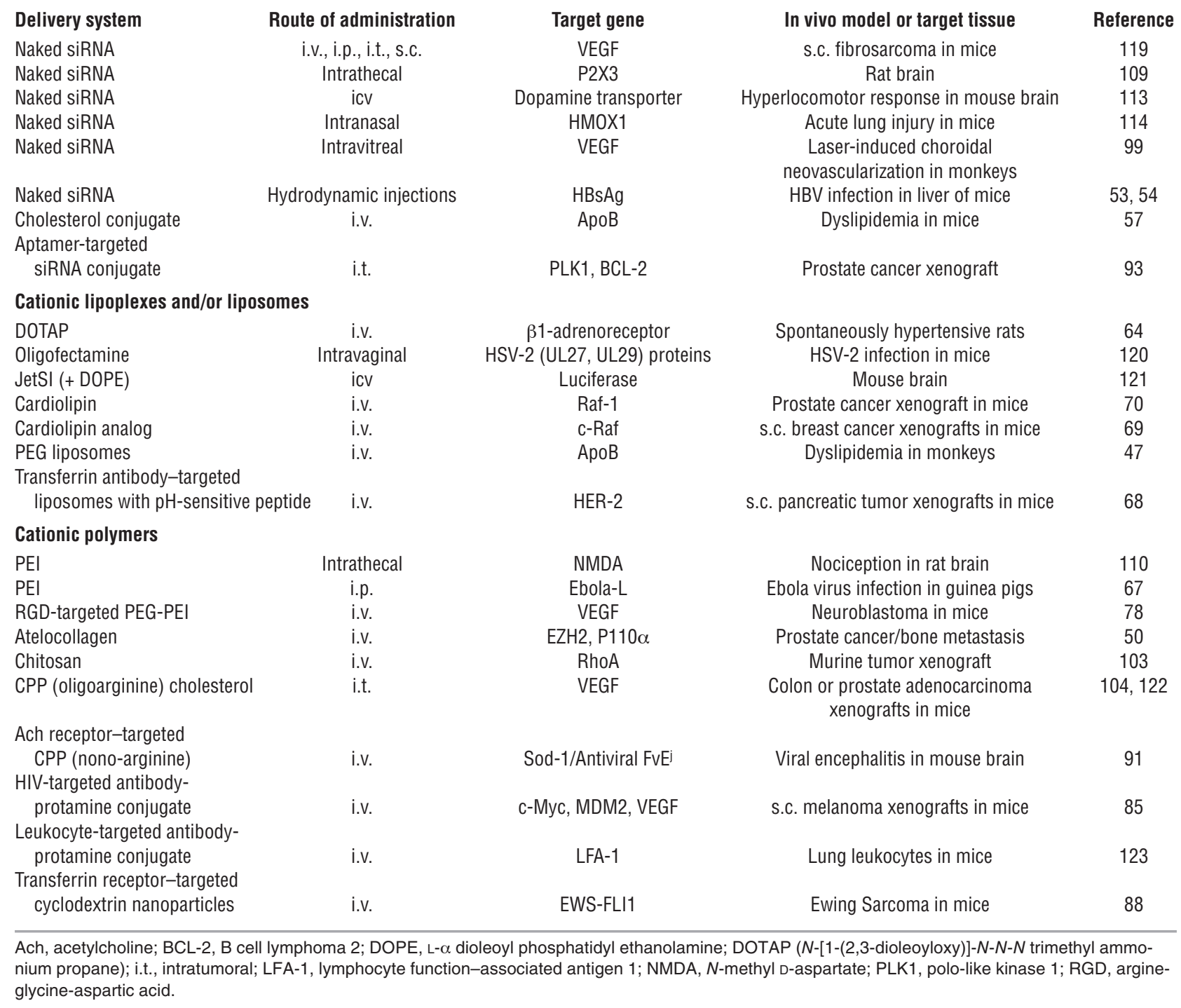

\section{Nonviral delivery strategies for siRNA in vivo}

Viral vectors are highly efficient delivery systems for nucleic acids, but their clinical application is hindered by their induction of toxic immune responses and inadvertent gene expression changes following random integration into the host genome, and as such, they are not covered further here (see instead ref. 10).

A number of nonviral siRNA delivery approaches that have now been reported in vivo, including in nonhuman primates and humans (see Clinical trials with siRNA therapentics), support the therapeutic development of siRNA for a number of different disease states (2-11). Of primary consideration in deciding on a drug delivery system for siRNA is whether the intended disease target lends itself to systemic or local administration, such as for superficial tumors, skin lesions, and mucosal tissue. Advantages of local administration include that the siRNA might only require simple formulation and thereby be easier to produce and administer, that it might facilitate site-specific delivery for localized effects, and that it is likely to require lower doses for efficacy that exert minimal systemic effects with a reduced risk of off-target effects, and thus it is likely to be the most cost-efficient strategy for siRNA delivery in vivo. A particular advantage of systemic delivery is that it is more widely applicable to the clinic, where more diseases require i.v. or i.p. injection treatment regimens. Of course, the holy grail of delivery strategies is finding a way of delivering siRNA orally, but this has not been investigated in much detail as yet, probably due to the major hurdle of siRNA degradation and likely poor bioavailability from the gastrointestinal tract.

\section{In vivo biodistribution of siRNAs administered systemically}

It is important that exogenously administered siRNAs accumulate at their target tissues and organs so as to exert their biological action at these sites with minimal effects on nontarget tissues. Although route of administration is an important variable, systemically administered naked nucleic acids generally accumulate in the organs of the reticuloendothelial system (RES) such as the 
liver, lung, spleen, and kidneys (43), and recent reports suggest that siRNAs also behave similarly (44-46). Braasch et al. (44) first showed that siRNAs in mice accumulate in the liver and kidneys following systemic administration. Additional studies $(45,46)$ also showed that naked siRNAs accumulate in the kidneys of mice and are detectable in the urine as early as 5 minutes after i.v. injection. Taken together, these studies suggest that siRNAs behave as typical macromolecules of less than $50 \mathrm{kDa}$ and $6 \mathrm{~nm}$, in that they are susceptible to glomerular filtration in the kidney and excretion in the urine.

The use of siRNA delivery strategies such as conjugation to polyethylene glycol (PEGylation) or formation of complexes with lipids or polymers (Table 1) prevents excretion in the urine, as these formulation constructs exceed the molecular weight and size range for glomerular filtration. Thus the type of siRNA delivery system used seems to be important for defining pharmacokinetics and biodistribution as well as the maximum tolerated doses. For example, Zimmermann et al. (47) reported that a nontargeted lipidformulated siRNA exhibited no toxicities at a dose of $1 \mathrm{mg} / \mathrm{kg}$, whereas a dose of $2 \mathrm{mg} / \mathrm{kg}$ resulted in substantial hepatotoxicity, suggesting that for this formulation, a $2-\mathrm{mg} / \mathrm{kg}$ dose of siRNA was near or above the maximum tolerated in cynomolgus monkeys. In contrast, doses of up to $9 \mathrm{mg} / \mathrm{kg}$ of siRNA formulated as a transferrin receptor-targeted cyclodextrin nanoparticle were well tolerated in cynomolgus monkeys (48). Hepatotoxicity and renal toxicity, as well as a mild immune response, were noted at a dose of $27 \mathrm{mg} / \mathrm{kg}$ with this formulation, but it was difficult to assess the precise maximal tolerated dose of siRNA, as there were no data for doses between $9 \mathrm{mg} / \mathrm{kg}$ and $27 \mathrm{mg} / \mathrm{kg}$. Thus delivery systems can be used to favorably alter the pharmacokinetics of naked siRNA and reduce the dosing requirements so as to minimize toxicity and possibly off-target effects and immune stimulation, both of which are often dose dependent.

Many of the early in vivo studies relied on the passive accumulation of siRNA following systemic administration to target gene silencing to the liver, kidney, lung, and heart - another organ benefitting from passive siRNA accumulation $(17,18)$. Furthermore, several in vivo studies have shown that siRNAs can also accumulate to some extent within s.c. tumors in nude mice (refs. 49, 50, and see below). This passive biodistribution might be due, in part, to the leaky vasculature of the tumors (51).

\section{Systemic delivery strategies in vivo}

Various strategies for delivering siRNA to specific tissue and organ systems in vivo following systemic administration are summarized in Table 1 and discussed here.

Hydrodynamic i.v. injection. Hydrodynamic i.v. injection involves the rapid i.v. injection of siRNA in large volumes of physiological buffer to achieve effective localization of duplex siRNA mainly in the liver, although distribution to the kidney, lung, and pancreas has been reported (52). Effective gene silencing in the liver of mice has been demonstrated using this delivery strategy with both unmodified and chemically modified siRNAs (53-55). Although the precise mechanisms of how siRNA is delivered to cells by hydrodynamic injection are unclear (56), it is probable that the high pressure causes membrane perturbations that facilitate siRNA uptake in vivo by some, but not all, cell types, even though they all might show membrane disruption (52). However, this delivery method cannot be directly translated to clinical use due to both the large injection volumes needed and the cellular toxicities caused by the high pressure of the injection.
Cholesterol conjugates of siRNA. Conjugation to lipophilic groups can enhance cell uptake and favorably improve pharmacokinetics and tissue biodistribution of oligonucleotides, and this strategy has now been applied to siRNA delivery. In mice, administration of an ApoB-specific siRNA with a chemically modified backbone conjugated to a lipophilic cholesterol moiety at the $3^{\prime}$ end of the sense strand resulted in knockdown of apoB mRNA by approximately $60 \%$ in the liver and approximately $75 \%$ in the jejunum (57). Surprisingly, no off-target effects or immune stimulation were reported despite the high 50-mg/kg dose needed for efficacy (57). However, extensive cell surface binding of lipophilic oligonucleotide conjugates has been reported to interfere nonspecifically with normal cellular processes such as dipeptide transport (58), necessitating detailed safety evaluation of this potential approach to clinical siRNA delivery.

Cationic delivery systems. Several types of synthetic vectors have been investigated for gene-silencing applications, including the development of cationic lipids and/or liposomes, cationic polymers, cationic dendrimers (branch-like polymer structures), and cationic cell-penetrating peptides (CPPs) (2-8, 16-19). A common theme amongst these vectors is their net positive (cationic) charge, which facilitates both complex formation with the polyanionic nucleic acid and interaction with the negatively charged cell membrane. The siRNA delivery (or transfection) reagent complexes are often specifically referred to as lipoplexes, dendriplexes, and polyplexes, depending on whether the vector used is a cationic lipid, dendrimer, or polymer, respectively. Alternatively, these cationic lipids and polymers can form nanoparticles, in which the nucleic acid is entrapped within the body of the particle and not simply adsorbed onto the surface, usually via ionic interactions, as is the case with complexes. However, in the literature, the term nanoparticle is often interchangeably used for complexes, possibly to better reflect the nomenclature being used within the ongoing nanotechnology/nanomedicine revolution (59). Although the exact mechanism of delivery varies, these complexes and nanoparticles can, to varying degrees, provide siRNA stability within blood serum, avoid sequestration within the RES, and eventually reach target cells. They are taken into the cell by some form of vesicular transport (endocytosis and/or macropinocytosis). Complexes and nanoparticles generally have to be less than $100 \mathrm{~nm}$ to avoid renal excretion and be taken up by cells (60). Once inside cells, the inclusion of fusogenic lipids such as L- $\alpha$ dioleoyl phosphatidyl ethanolamine (DOPE) and/or $\mathrm{pH}$-sensitive peptides such as poly-histidine-lysine in siRNA delivery systems are thought to help destabilize endosomal membranes to aid their release into the cytosol (60). Further surface modification of complexes or nanoparticles with PEG can stabilize nanoparticles and prolong circulation times, and attachment of a targeting ligand or antibody for a cell-specific receptor or antigen can enable tissue or cell type targeting of siRNA (Figure 2 and see below).

Cationic liposomal delivery systems. Although neutral liposomes have been successfully used to deliver siRNA in vivo (61), cationic liposomes are more commonly used for siRNA delivery. Commercially available cationic lipid formulations such as lipofectin, RNAifect (Qiagen), Oligofectamine, Lipofectamine (Invitrogen), and TransIT TKO (Mirus) have all been investigated as potential enhancers of siRNA delivery in vitro (16-18).

DOTAP ( $N$-[1-(2,3-dioleoyloxy)]- $N-N-N$ trimethyl ammonium propane) and Oligofectamine were some of the first lipid formulations to be examined for the in vivo delivery of siRNA and 


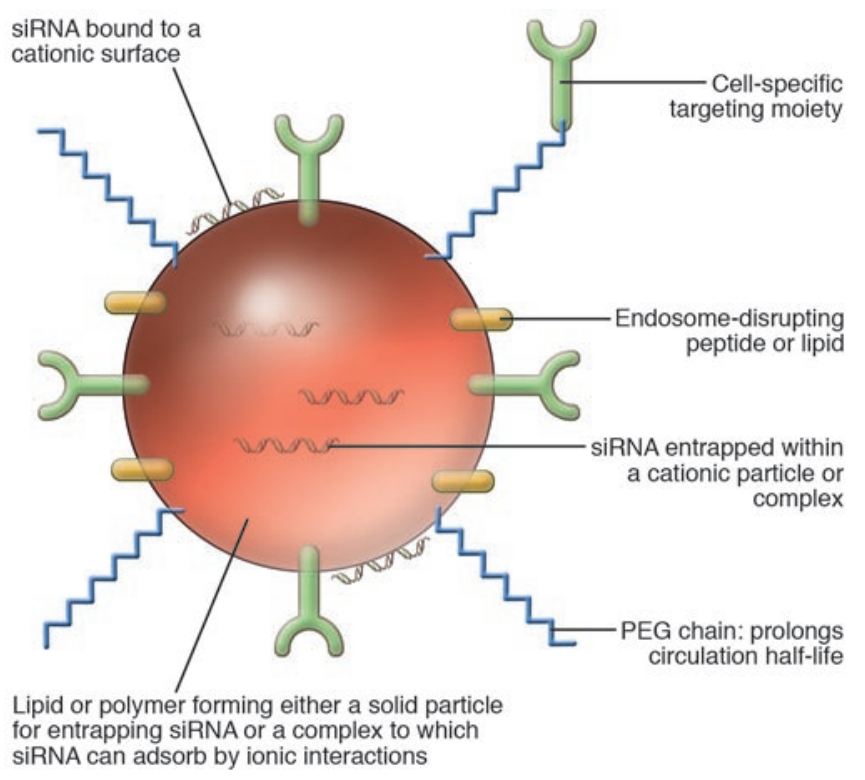

caused marked knockdown of TNF- $\alpha$ and $\beta$-catenin in mice (62, 63). Recently, Arnold et al. (64) showed that a single 1-mg/kg i.v. dose of siRNA-DOTAP lipoplexes selectively inhibited $\beta 1$-adrenoreceptor expression and markedly reduced blood pressure for up to 12 days. Interestingly, this sustained effect was greater than that obtained by just blocking the $\beta 1$-adrenoreceptor using $\beta$-blockers. It is not clear how long the siRNA complexes remained in vivo, as this was not reported in the study, but fluorescent microscopy revealed good biodistribution in the heart, lung, kidney, and spleen 3 hours after administration (64).

Cationic liposomes termed "solid nucleic acid lipid particles" (SNALPs) that have been stabilized by PEGylation for improved pharmacokinetics have also successfully been used to deliver siRNA systemically to silence the $a p o B$ gene in mice and nonhuman primates (47). A single dose of $2.5 \mathrm{mg} / \mathrm{kg}$ of siRNA encapsulated within the SNALP formulation reduced $a p o B$ gene expression in the livers of cynomolgus monkeys by more than $90 \%$. This led to substantial reductions in serum cholesterol and LDL that were sustained for more than 11 days after dosing, implying that this delivery system provides sustained delivery of siRNA to the liver (47), although alternative sustained release formulations such as poly (D,L-lactide-co-glycolide) (PLGA) copolymer microspheres have also been investigated for siRNA delivery (65). The passive accumulation of SNALPs in the liver has also been taken advantage of for the potential treatment of experimental hepatitis B (66) and infection with Ebola virus (67). Targeting of other organs with SNALPs is likely to require the attachment of a targeting ligand or antibody.

Pirollo et al. (68) demonstrated that cationic liposomes bearing a transferrin receptor-specific single-chain antibody fragment effectively delivered a HER2-specific siRNA to tumor xenografts in nude mice. In vitro antitumor effects, as evidenced by a 3-fold greater sensitivity to chemotherapeutic agents, were further enhanced by the inclusion of a $\mathrm{pH}$-sensitive histidine-lysine peptide into the nanoparticle complexes (68). Presumably, the peptide facilitated endosomal release of naked and chemically modified siRNA, although this was not obvious from the similar intracellular biodistributions reported for the complexes with and without the peptide (68). It was also unclear whether the $\mathrm{pH}$-sensitive peptide truly enhanced endosomal

\section{Figure 2}

A schematic depiction of the structural components required of a targeted cationic complex or nanoparticle for siRNA delivery in vivo. Ordered assembly of the complex or nanoparticle $(\sim 100 \mathrm{~nm}$ in size) typically involves the use of a biocompatible/genocompatible lipid or polymer core that enters tissues and cells readily. The siRNA can be either entrapped within the core, adsorbed onto the surface via ionic interactions, or covalently attached through the sense strand to one of the surface components. The particle can then be modified to bear PEG polymer chains that improve the pharmacokinetic and biodistribution behavior by prolonging half-lives in vivo. PEGylation also serves to improve the biocompatibility of the particles and, because of shielding of the negative charges, to decrease nonspecific interactions with extracellular matrix components. Targeting to a specific tissue or cell type can be facilitated by addition of a receptor ligand or antibody that may be attached to the surface or conjugated to the PEG. Following uptake by endocytosis, intracellular bioavailability can be enhanced through endosomal escape of siRNA by including a fusogenic lipid or $\mathrm{pH}$-sensitive peptide in the complex or nanoparticle (see text for specific examples).

efflux and activity of siRNA in vivo, as a direct comparison of the two delivery platforms was not reported in the same in vivo study (68).

There is no denying that cationic lipid nanoparticles and complexes with siRNA are effective when delivered systemically. However, experience with antisense oligonucleotides and ribozymes $(12,13)$ suggests their widespread use clinically might be tempered somewhat by toxicological concerns. Cationic lipid-mediated cellular toxicity has been observed in vitro and in vivo (16-18), and more recently it has been shown in vitro that some cationic lipid formulations elicit inadvertent gene expression (38) and enhance the immune response to siRNA (33). Development of novel serum stable, non-toxic lipids is needed for clinical delivery of siRNA, and recent attempts at using natural lipid analogs of cardiolipin have been successful in delivering siRNA systemically to cancer xenografts $(69,70)$. It should be noted that PEGylated liposomes are a clinically approved delivery system for doxorubicin and therefore represent a viable option for delivering siRNA in humans. However, care has to be taken with repeated dosing regimens because acute hypersensitivity due to the generation of antibodies specific for PEGylated particles has been reported $(71,72)$, but this might be circumvented by pretreatment with conventional drugs.

Cationic polymer and peptide delivery systems for siRNA. Linear or branched cationic polymers including peptides readily bind and condense DNA and have thus been widely used as transfection reagents for genes, oligonucleotides, and now duplex siRNA (12, 13, 16-19). The high charge density of cationic polymers allows them to escape from endosomes and deliver their nucleic acid cargo into the cytosol through the so-called "proton-sponge" effect. The polycationic nature of the polymer is thought to buffer low endosomal $\mathrm{pH}$ through enhanced influx of protons and water, culminating in endosome rupture (16-18). Of the many cationic polymers, polyethyleneimine (PEI) has been widely studied for DNA, oligonucleotide, and siRNA delivery (6). PEI is available in either linear or branched form and in many molecular weights, ranging from $1 \mathrm{kDa}$ to more than $1,000 \mathrm{kDa}$. Generally, lowmolecular-weight PEIs ( $<25 \mathrm{kDa})$ with a branched rather than linear architecture are thought to be superior transfection reagents, as higher molecular weights tend to be toxic $(6,73,74)$. 
siRNAs delivered by PEI have been effective antiviral agents in a murine model of influenza infection (75) and a guinea pig model of Ebola virus infection (67). PEI also effectively delivered siRNA targeting HER2 in mouse models of ovarian carcinomas (76). Thus PEI seems to be a promising siRNA delivery system for systemic use, provided the correct architecture and molecular weight polymer are selected so as to avoid toxicities. Microarray analysis of tumors treated by linear and branched PEI revealed that both polymer architectures induced multiple gene changes in vivo that might contribute to off-target effects of siRNA and also have an impact on gene-silencing activity (18).

Arg-Gly-Asp (RGD) peptides have been used to target drugs and imaging agents to tumor vasculature expressing $\alpha_{v} \beta_{3}$ integrin, and an RGD-targeted radiotracer has successfully been tested in humans for the visualization of $\alpha_{v} \beta_{3}$ integrin, which demonstrates the feasibility of this approach (77). Now, a novel delivery strategy using PEGylated PEI with an RGD peptide to deliver siRNA targeting VEGF has been shown to inhibit tumor growth and reduce angiogenesis after i.v. administration (78).

Another class of polymers widely used for nucleic acid transfection are the polyamidoamines $(79,80)$. Although the commercially available polyamidoamine polymers Polyfect and Superfect (both from Qiagen) are dendrimers that were initially designed for the delivery of plasmids, they have also now been used for siRNA delivery in vitro $(24,81-83)$ and might be used in vivo. Advantages of dendrimer delivery systems are that they can be engineered to have precise molecular weights and defined molecular sizes and shapes for avoiding biological barriers in vivo. However, nonspecific toxicities, including induction of pleiotropic gene expression, might hinder their progress in vivo (24).

The cationic polyamine, protamine, can complex with and condense nucleic acids and therefore has long been used for intracellular delivery of plasmid DNA and other oligonucleotides (84). Recently it has been incorporated into antibody conjugates for delivering siRNA to tumors in vivo. Song et al. (85) used a conjugate of a fragment antibody (Fab) specific for the HIV-1 envelope protein gp160 and protamine, to which the siRNA could complex. siRNAs selectively delivered by Fab-protamine conjugates to tumor cells engineered to express gp160 had a marked antitumor effect in vivo.

Atelocollagen is a very large protein $(300 \mathrm{kDa})$ derived from pepsintreated type I collagen from calf dermis that has been used to administer siRNA systemically and locally in tumor models $(49,50,86,87)$. When complexed with siRNA, this cationic polyamine improved the half-life of naked siRNA by 3 -fold and increased cell uptake and activity comparable to the cationic liposome JetSI (87). Atelocollagen also seemed to function as a sustained release delivery vehicle in vitro and in vivo $(49,50,87)$. In vivo, this polymer was also able to effectively deliver siRNA targeting VEGF to tumor vasculature in a xenograft model of prostate cancer (49), to bone metastases (50), and to an orthotopic model of human testicular cancer (87).

A cyclodextrin polycation delivery system that seems to mask immune stimulation effects of siRNA, even siRNAs with known immunostimulatory sequences, has been described $(48,88)$. It consists of a cyclodextrin core to which is attached PEG and transferrin - a tumor-targeting ligand. Using this delivery system, effective gene silencing of the oncogene EWS-FLI1 was reported in a metastatic model of Ewing sarcoma (88). More recently, a multidosing study of this delivery system in cynomolgus monkeys has suggested that the delivery system is well tolerated and that even repeat doses failed to elicit a significant delivery system-specific antibody response (48).
CPPs, also known as protein transduction domains and membrane transduction sequences, are short cationic peptide chains with a maximum of 30 amino acids that have been used for siRNA delivery in vitro (89). Although not as potent as cationic lipid delivery agents in vitro, CPP-oligonucleotide conjugates offer the advantage of being molecules rather than particles and might have substantial advantages over particle-based delivery in vivo (90). This was highlighted in a recent study that reported for the first time that systemically delivered siRNA-peptide conjugates can cross the blood-brain barrier (BBB) (91). Other approaches being considered for systemic delivery to the brain include the use of transferrin receptor antibody-targeted PEGylated liposomes that might selectively bind to the vasculature of the BBB, which expresses high levels of the transferrin receptor (92).

Other delivery systems. Other in vivo delivery approaches for siRNAs include aptamer-siRNA conjugates that specifically inhibit tumor growth and mediate tumor regression in a xenograft model of prostate cancer (93) and the use of a chitosan-based siRNA nanoparticle delivery system to knockdown EGFP both in vivo and in vitro (94). Furthermore, siRNA delivery to the alveoli of mice could be achieved by using lung surfactant, a lipoprotein that normally lines the lung wall (95).

Studying uptake and delivery mechanisms with novel nanoparticle approaches. It is important to understand the detailed mechanism and biodistribution of siRNA and its delivery vectors to further optimize and develop new formulations for use in the clinic. In this regard, Medarova et al. (96) have reported a new dual-purpose nanoparticle probe for the simultaneous delivery and imaging of siRNA in tumors following systemic administration. The produced supramagnetic nanoparticles were coated with aminated dextrans labeled with a fluorescent dye and, via two different linkers, attached to an active siRNA and a CPP to facilitate cell uptake. Localization of the nanoparticles to experimental tumors in mice was observed using both MRI and optical imaging techniques. Effective delivery and gene silencing was also demonstrated with a survivin-specific siRNA in nude mice bearing s.c. colorectal carcinoma xenografts (96). Interestingly, even in the absence of specific targeting, the probe accumulated in tumors following i.v. injection (96). This was explained by the possible leakiness of the tumor vasculature, which could facilitate enhanced permeability and retention of macromolecules and nanoparticles (51). The study also highlighted that covalent attachment of siRNA to particles does not impair gene-silencing activity, something that bodes well for further developments in this field.

Toxicogenomics and genocompatibility of in vivo delivery systems. Optimal systemic delivery should be achieved without compromising siRNA gene-silencing activity and specificity. It has traditionally been thought that drug delivery systems used in siRNA experiments are biologically and "genomically" inert $(18,97,98)$. However, recent microarray-based gene expression studies have shown that this is not the case and that transfection reagents such as cationic lipids and cationic polymers can directly induce gene expression changes in biological systems that might have an impact on siRNA activity and specificity $(18,24,38,39)$. This phenomenon occurred both in addition to and separately from the ability of some delivery systems to enhance the immune response of siRNA (33). Direct intratumoral administration of PEI to epithelial tumor xenografts in nude mice revealed that branched PEI had a greater propensity than the linear form to induce gene changes in vivo and this correlated well with the reported greater toxicity of branched PEI (18). Interestingly, two polyamidoamine dendrimers, differing 
only in their structural architecture, elicited many different gene expression changes in human cells, including opposing effects on the level of EGFR expression despite equivalent levels of siRNA uptake (24). This led to either a marked enhancement or attenuation of the gene-silencing effect with EGFR-specific siRNA, thereby suggesting that gene changes induced by delivery reagents, separate from the effects on cellular uptake, are important in determining the level of gene silencing achieved by siRNAs.

Delivery system-induced effects on gene expression might be exploited to enhance siRNA gene-silencing activity by selecting delivery systems that also inhibit target gene(s). Conversely, delivery systems that enhance target gene expression should be avoided, as they can compromise gene-silencing activity of siRNA. Any beneficial effects on siRNA potency, however, need to be balanced against any increase in off-target effects due to the delivery system. For this reason, it has been advocated that delivery systems should be screened for "genocompatibilty" so that they do not markedly increase off-target effects and/or adversely increase expression of the target gene(s) in siRNA gene-silencing studies $(18,24)$.

A natural extrapolation of these studies is that a delivery system might be engineered to have any desirable property to act in concert with siRNA therapy for many different pathologies. Therefore it opens the door for synthetic chemists to engineer new vectors with desirable intrinsic biological activity (e.g., to have antitumor activity) or genomic activity (e.g., to downregulate the same target gene as the siRNA). If successful, such targeted delivery system designs might have a significant impact on broadening RNAimediated gene-silencing applications in the clinic.

\section{Local delivery strategies in vivo}

A number of studies have been published recently that make use of local delivery of siRNA to a number of diverse organs, and several have served as proof-of-concept for the current clinical trials with siRNA (see Clinical trials with siRNA therapentics).

Intraocular delivery. Delivery of siRNA to the eye has been successful in the form of intravitreal injections in cynomolgus monkeys (99) and subretinal injections in mice (100). In both studies, siRNA targeting VEGF was used to prevent laser-induced choroidal neovascularization in an experimental model of age-related macular degeneration (AMD). In the nonhuman primates, the effect of a single injection of siRNA persisted for at least 36 days (99). In mice, subretinal injection of siRNA 36 hours after laser treatment resulted in a $75 \%$ reduction in neovascularization when compared with control (100). The delivery of siRNA using subconjunctival injections has also been successful (101) - 200-nM siRNA targeting TGF- $\beta$ complexed with TransIT TKO lipid formulation (Mirus) led to an approximately $50 \%$ decrease in inflammatory cell infiltration 2 days after injection. Herard et al. (102) showed that 24 hours after intraocular injection, siRNA targeting amyloid precursor protein (APP) accumulated in retinal cells, and substantial knockdown of APP in retinal terminals was observed. Therefore local delivery to the eye of naked and lipid-complexed siRNA is possible, and such studies have paved the way for human clinical trials (see Clinical trials with siRNA therapentics).

Intratumoral delivery. The direct local injection of siRNAs, with or without a delivery system, into tumors has been an effective anticancer strategy in vivo $(96,103,104)$. For example, tumor volume was reduced by $85 \%$ and $53 \%$ when siRNA targeting Ras $A$ and Ras $C$, respectively, was repeatedly administered to mice in a xenograft breast cancer model (103). Recently, a cholesterol-modified low- molecular-weight PEI has been used to locally deliver siRNA targeting VEGF to tumor xenografts in vivo (104), and intratumoral delivery of PEI-complexed plasmids targeting FAK markedly suppressed primary tumor growth and metastasis of B16F10 cells into lung and lymph nodes (105). Both approaches prolonged the survival of the tumor-bearing mice. However, clinically, only a few tumors are amenable to local delivery, and systemic approaches such as those described above are necessary for most solid tumors in vivo.

Local in vivo electroporation to muscle. Electroporation, a technique whereby electrical currents are used to gently make cell membranes permeable to macromolecules, has been used to deliver siRNA to mouse muscle tissue $(106,107)$. For example, Golzio et al. (107) targeted GFP expressed exogenously from a plasmid in mouse muscles. When siRNA was cotransfected with the plasmid encoding GFP, a substantial silencing effect was noted for up to 23 days. However, the approach has obvious safety concerns relating to the application of electrical charge to sensitive tissues, although its safe use in the CNS of rats has been reported (108).

Local delivery to the CNS. The CNS is a region that is difficult to deliver drugs to due to the BBB. The treatment of diseases affecting the CNS is therefore often inadequate, for example in the case of brain tumors. Thus siRNA is of potential benefit in diseases for which there are limited treatment options. As naked siRNA duplexes are unable to cross the $\mathrm{BBB}$, local delivery methods have been employed for gene silencing in the CNS (92). For example, naked siRNA has been reported to effect gene silencing when delivered locally to the CNS of either rats or mice following intrathecal infusions $(109,110)$, bilateral intra-arcuate injections to the hypothalamus (111), injections to the brain stem (112), and intracerebroventricular infusion to the dorsal third ventricle (113). For example, PEI-siRNA polyplexes targeting the NR2B subtype of the NMDA receptor that were administered via intrathecal injection reduced protein levels by $83 \%$ after 7 days in the rat brain (110). This led to a reduced response to the continual pain induced by formalin (110).

Local delivery of siRNA to the visual cortex and hippocampal regions of rat brains using an electroporation technique has also been reported (108), implying that the technique is well tolerated on the delicate tissues of the brain. Infusion to the third dorsal ventricle resulted in knockdown of the targeted dopamine transporter in areas of the brain distant from the site of injection (113). A significant reduction of dopamine transporter was reported in all the brain sections tested, and an increase in expected behavioral changes was observed in siRNA-treated animals (113).

Taken together, these studies suggest that local delivery of siRNA to the CNS is a feasible option for targeting CNS-based diseases, though systemic delivery strategies may be clinically more attractive $(91,92)$.

Intranasal delivery to the lung. Intranasal delivery is a technique that is widely used to deliver drugs to the lungs. Delivering siRNA to the lung could be a potential treatment for a number of diseases including asthma, cystic fibrosis, ischemic reperfusion injury, and infection with respiratory viruses. Delivery of siRNA has been achieved in vivo by using intranasal techniques $(94,114,115)$. The role of heme oxygenase-1 (HO-1) in lung reperfusion injury was examined by anesthetizing mice and administering siRNA intranasally (116). After initiation of ischemic reperfusion injury, levels of HO-1 in animals treated with siRNA did not rise (unlike in control animals), and this was associated with apoptosis. siRNA delivered by this method had no effect in other organs that were tested. In contrast, knockdown of GAPDH by siRNA was observed in organs other than the lung 
Table 2

Examples of ongoing or planned clinical trials for siRNA-mediated RNAi

\begin{tabular}{lccc} 
Company & siRNA product & Disease & Status \\
Acuity Pharmaceuticals & Cand5 & AMD & phase II \\
& Cand5 & Diabetic macular edema & Phase II \\
Alnylam & ALN-RSV01 & RSV lung infection & Phase II \\
& Unknown & Pandemic flu & Preclinical \\
& ALN-VSP01 & Liver cancer & Preclinical \\
Silence Therapeutics & RTP-801i & AMD & Phase I \\
& AKli-5 & Acute renal injury & Preclinical \\
& Atu027 & Gastrointestinal cancers & Preclinical \\
Sirna Therapeutics/Merck & Atu093 & Non-small cell lung cancer & Preclinical \\
& Sirna-027 & AMD & Phase I \\
& Sirna-034 & HCV & Preclinical \\
& & & \\
\hline
\end{tabular}

safety, tolerability, and pharmacokinetics in healthy adult volunteers. A total of 100 human subjects received ALN-RSV01 either as a single dose or as multiple daily doses in the form of a nasal spray. ALNRSV01 was found to be safe when administered in relevant doses, with a mild adverse event profile comparable with placebo. There was no evidence of electrocardiographic abnormalities in subjects exposed to the drug and no significant systemic exposure to the ALN-RSV01 that was administered intranasally. Based on these results, and as of June 2007, Alnylam has initiated a phase II trial with ALN-RSV01 that is designed to evaluate the safety, tolerability, and antiviral activ-

following intranasal delivery (94). Several other studies also show that intranasal delivery to the lung leads to effective gene silencing in acute models of lung injury (117) and in the treatment of experimental infection with SARS virus in nonhuman primates (118).

Bitko et al. (115) delivered siRNA targeting respiratory syncytial virus (RSV) and parainfluenza virus intranasally to anesthetized mice with and without the lipid delivery system TransIT TKO (Mirus). This resulted in almost complete elimination of the virus when siRNA was delivered using TransIT TKO, although siRNA alone also produced a marked decrease in pulmonary viral titer. The administration of siRNA did not produce an IFN response, and no adverse effects of the delivery system were noted. A marked difference in body weight gain and in viral titer was noted when administration of siRNA was delayed after infection by differing times; the length of delay was inversely proportional to the efficacy of the siRNA (115). Therefore, it seems that siRNA has benefits for acute treatment of pulmonary disease and that lipid carriers are well tolerated when used intranasally.

\section{Clinical trials with siRNA therapeutics}

There are several clinical trials ongoing or planned for taking siRNA into the clinic in the treatment of important diseases such as macular degeneration, cancer, and respiratory diseases (Table 2).

The first ever human clinical trial with siRNA was conducted by Acuity Pharmaceuticals in late 2004 in patients suffering from AMD. Local intravitreal delivery of siRNA (Cand5) targeting VEGF was designed to prevent the overgrowth of new blood vessels in the "wet" form of AMD. This molecule has now reached phase II clinical evaluation, with preliminary results indicating that there are dose-related benefits with respect to several clinical endpoints such as near vision and lesion size. Cand5, as of early 2006, is also being evaluated in a separate phase II trial for efficacy in diabetic macular edema. Sirna Therapeutics, now owned by Merck, entered their first siRNA (Sirna-027) targeting VEGFR for the treatment of AMD. Preliminary data from the trial suggested that Sirna-027 administered as a single intravitreal injection at doses up to $800 \mu \mathrm{g}$ was well tolerated by patients and improvements in the visual acuity of a subset of subjects has also been observed. Silence Therapeutics (formerly Atugen) also has entered RTP-801i siRNA into phase I trials for AMD and is planning trials with another siRNA for the treatment of acute renal injury (Table 2).

Alnylam Pharmaceuticals recently completed two phase I clinical trials with their siRNA targeting RSV, ALN-RSV01, to evaluate its ity of ALN-RSV01 in about 90 adult subjects experimentally infected with RSV. These favorable early reports and rapid development auger well for further clinical trials with siRNA.

\section{Concluding remarks}

In conclusion, siRNA has quickly been established as a robust and effective gene-silencing strategy in animal models, and more recently in human clinical trials, as a potential therapeutic approach. Its current success and future development will clearly benefit from the scientific experiences acquired from related gene-silencing technologies such as antisense oligonucleotides, ribozymes, and DNAzymes. However, future research needs to address the important challenges relating to more effective design, enhanced biological stability, and efficient targeted delivery in vivo.

A number of important lessons can be learned from the seemingly disparate in vivo studies with siRNA. Delivery systems designed to favorably alter the pharmacokinetics and biodistribution of siRNAs are essential for systemic use, as they prolong siRNA halflives in serum and blood and reduce the effective dose of naked siRNA. At present, passive targeting of siRNA is limited to the RES and the leaky vasculature of some tumors, and therefore robust targeting strategies are needed for targeting deeper tissues and organs for clinical purposes. As we learn more about the in vivo actions of siRNA delivery systems, including their toxicogenomics and biodistribution, it will be possible to refine our delivery strategies for improved targeting and specificity of these molecules for any given clinical condition. It is envisaged that ultimately the development of safer siRNA targeted delivery systems that are biocompatible and genocompatible, in that they avoid immune stimulation and off-target gene effects, will lead to an expansion in the clinical roles of this Nobel prize-winning technology.

\section{Acknowledgments}

Research projects on siRNA in the laboratory of S. Akhtar while at Cardiff University (Cardiff, United Kingdom) were funded by CRUK, Biotechnology and Biological Sciences Research Council, and the British Heart Foundation. The authors acknowledge funding from Kuwait University.

Address correspondence to: Saghir Akhtar, Professor in Pharmacology and Toxicology, Faculty of Medicine, Health Sciences Center, Kuwait University, PO Box 24923, Safat 13110, Kuwait. Phone: $965-$ 4986342; Fax: 965-5342583; E-mail: SA_Pharma@hotmail.co.uk. 
1. Fire, A., et al. 1998. Potent and specific genetic interference by double-stranded RNA in Caenorhabditis elegans. Nature. 391:806-811.

2. Bumcrot, D., Manoharan, M., Koteliansky, V., and Sah, D.W. 2006. RNAi therapeutics: a potential new class of pharmaceutical drugs. Nat. Chem. Biol. 2:711-719.

3. Kuhn, R., Streif, S., and Wurst, W. 2007. RNA interference in mice. Handb. Exp. Pharmacol. 178:149-176.

4. de Fougerolles, A., Vornlocher, H.P., Maraganore, J., and Lieberman, J. 2007. Interfering with disease: a progress report on siRNA-based therapeutics. Nat. Rev. Drug Discov. 6:443-453.

5. Kim, D.H., and Rossi, J.J. 2007. Strategies for silencing human disease using RNA interference. Nat. Rev. Genet. 8:173-184.

6. Aigner, A. 2006. Delivery systems for the direct application of siRNAs to induce RNA interference (RNAi) in vivo. J. Biomed. Biotechnol. 2006:71659.

7. Leung, R.K., and Whittaker, P.A. 2005. RNA interference: from gene silencing to gene-specific therapeutics. Pharmacol. Ther. 107:222-239.

8. Xie, F.Y., Woodle, M.C., and Lu, P.Y. 2006. Harnessing in vivo siRNA delivery for drug discovery and therapeutic development. Drug Discov. Today. 11:67-73.

9. Aagaard, L., and Rossi, J.J. 2007. RNAi therapeutics: principles, prospects and challenges. Adv. Drug Deliv. Rev. 59:75-86.

10. Martin, S.E., and Caplen, N.J. 2007. Applications of RNA interference in mammalian systems. Annu. Rev. Genomics Hum. Genet. 8:81-108.

11. Liu, G., Wong-Staal, F., and Li, Q.X. 2007. Development of new RNAi therapeutics. Histol. Histopathol. 22:211-217.

12. Akhtar, S., et al. 2000. The delivery of antisense therapeutics. Adv. Drug Deliv. Rev. 44:3-21.

13. Hughes, M.D., Hussain, M., Nawaz, Q., Sayyed, P., and Akhtar, S. 2001. The cellular delivery of antisense oligonucleotides and ribozymes. Drug Discov. Today. 6:303-315

14. Martinez, J., Patkaniowska, A., Urlaub, H., Luhrmann, R., and Tuschl, T. 2002. Single-stranded antisense siRNAs guide target RNA cleavage in RNAi. Cell. 110:563-574.

15. Parker, J.S., and Barford, D. 2006. Argonaute: a scaffold for the function of short regulatory RNAs. Trends Biochem. Sci. 31:622-630.

16. Gilmore, I.R., Fox, S.P., Hollins, A.J., Sohail, M., and Akhtar, S. 2004. The design and exogenous delivery of siRNA for post-transcriptional gene silencing. J. Drug Target. 12:315-340.

17. Gilmore, I.R., Fox, S.P., Hollins, A.J., and Akhtar, S. 2006. Delivery strategies for siRNA-mediated gene silencing. Current Drug Deliv. 3:147-155.

18. Akhtar, S., and Benter, I. 2007. Toxicogenomics of non-viral drug delivery systems for RNAi: Potential impact on siRNA-mediated gene silencing activity and specificity. Adv. Drug Deliv. Rev. 59:164-182.

19. Kawakami, S., and Hashida, M. 2007. Targeted delivery systems of small interfering RNA by systemic administration. Drug Metab. Pharmacokinet. 22:142-51.

20. Vorhies, J.S., and Nemunaitis, J. 2007. Nonviral delivery vehicles for use in short hairpin RNAbased cancer therapies. Expert Rev. Anticancer Ther. 7:373-382.

21. Chang, K., Elledge, S.J., and Hannon, G.J. 2006. Lessons from nature: microRNA-based shRNA libraries. Nat. Methods. 3:707-714.

22. Esau, C.C., and Monia, B.P. 2007. Therapeutic potential for microRNAs. Adv Drug Deliv Rev. 59:101-114.

23. Hammond, S.M. 2006. MicroRNA therapeutics: a new niche for antisense nucleic acids. Trends $\mathrm{Mol}$. Med. 12:99-101.

24. Hollins, A.J., Omidi, Y., Benter, I.F., and Akhtar, S.
2007. Toxicogenomics of drug delivery systems: Exploiting delivery system-induced changes in target gene expression to enhance siRNA activity. J. Drug Target. 15:83-88.

25. Fedorov, Y., et al. 2005. Different delivery methods - different expression profiles. Nat. Methods. 2:241.

26. Jackson, A.L., et al. 2003. Expression profiling reveals off-target gene regulation by RNAi. Nat. Biotechnol. 21:635-637.

27. Fedorov, Y., et al. 2006. Off-target effects by siRNA can induce toxic phenotype. RNA. 12:1188-1196.

28. Jackson, A.L., et al. 2006. Widespread siRNA "offtarget" transcript silencing mediated by seed region sequence complementarity. RNA. 12:1179-1187.

29. Birmingham, A., et al. 2006. 3' UTR seed matches, but not overall identity, are associated with RNAi off-targets. Nat. Methods. 3:199-204.

30. Lim, L.P., et al. 2005. Microarray analysis shows that some microRNAs downregulate large numbers of target mRNAs. Nature. 433:769-773.

31. Lin, X., et al. 2005. siRNA-mediated off-target gene silencing triggered by a $7 \mathrm{nt}$ complementation. Nucleic Acids Res. 33:4527-4535.

32. Sledz, C.A., Holko, M., de Veer, M.J., Silverman, R.H., and Williams, B.R. 2003. Activation of the interferon system by short-interfering RNAs. Nat. Cell Biol. 5:834-839.

33. Judge, A.D., et al. 2005. Sequence-dependent stimulation of the mammalian innate immune response by synthetic siRNA. Nat. Biotechnol. 23:457-462.

34. Jackson, A.L., et al. 2006. Position-specific chemical modification of siRNAs reduces off-target transcript silencing. RNA. 12:1197-1205.

35. Schwarz, D.S., et al. 2003. Asymmetry in the assembly of the RNAi enzyme complex. Cell. 115:199-208

36. Khvorova, A., Reynolds, A., and Jayasena, S.D. 2003. Functional siRNAs and miRNAs exhibit strand bias [erratum 2003, 115:505]. Cell. 115:209-216.

37. Reynolds, A., et al. 2004. Rational siRNA design for RNA interference. Nat. Biotechnol. 22:326-330.

38. Omidi, Y., et al. 2003. Toxicogenomics of nonviral vectors for gene therapy: a microarray study of lipofectin- and oligofectamine-induced gene expression changes in human epithelial cells. J. Drug Target. 11:311-323.

39. Omidi, Y., Hollins, A.J., Drayton, R.M., and Akhtar, S. 2005. Polypropylenimine dendrimer-induced gene expression changes: the effect of complexation with DNA, dendrimer generation and cell type. J. Drug Target. 13:431-443.

40. Beltinger, C., et al. 1995. Binding, uptake, and intracellular trafficking of phosphorothioatemodfied oligodeoxynucleotides. J. Clin. Invest. 94:1814-1823

41. Beale, G., et al. 2003. Gene silencing nucleic acids designed by scanning arrays: anti-EGFR activity of siRNA, ribozyme and DNA enzymes targeting a single hybridization-accessible region using the same delivery system. J. Drug Target. 11:449-456.

42. Bartlett, D.W., and Davis, M.E. 2006. Insights into the kinetics of siRNA-mediated gene silencing from live-cell and live-animal bioluminescent imaging. Nucleic Acids Res. 34:322-233.

43. Akhtar, S., and Agrawal, S. 1997. In vivo studies with antisense oligonucleotides. Trends Pharmacol. Sci. 18:12-18.

44. Braasch, D.A., et al. 2004. Biodistribution of phosphodiester and phosphorothioate siRNA. Bioorg. Med. Chem. Lett. 14:1139-1143.

45. Santel, A., et al. 2006. A novel siRNA-lipoplex technology for RNA interference in the mouse vascularendothelium. Gene Ther. 13:1222-1234.

46. van de Water, F.M., et al. 2006. Intravenously administered short interfering RNA accumulates in the kidney and selectively suppresses gene function in renal proximal tubules. Drug Metab. Dispos.
34:1393-1397.

47. Zimmermann, T.S., et al. 2006. RNAi-mediated gene silencing in non-human primates. Nature. 441:111-114

48. Heidel, J.D., et al. 2007. Administration in nonhuman primates of escalating intravenous doses of targeted nanoparticles containing ribonucleotide reductase subunit M2 siRNA. Proc. Natl. Acad. Sci. U. S. A. 104:5715-5721.

49. Takei, Y., Kadomatsu, K., Yuzawa, Y., Matsuo, S., and Muramatsu, T. 2004. A small interfering RNA targeting vascular endothelial growth factor as cancer therapeutics. Cancer Res. 64:3365-3370.

50. Takeshita, F., et al. 2005. Efficient delivery of small interfering RNA to bone-metastatic tumors by using atelocollagen in vivo. Proc. Natl. Acad. Sci. U. S. A. 102:12177-12182.

51. Iyer, A.K., Khaled, G., Fang, J., and Maeda, H. 2006. Exploiting the enhanced permeability and retention effect for tumor targeting. Drug Discov. Today. 11:812-818.

52. Lewis, D.L., and Wolff, J.A. 2007. Systemic siRNA delivery via hydrodynamic intravascular injection. Adv. Drug Deliv. Rev. 59:115-123.

53. Giladi, H., et al. 2003. Small interfering RNA inhibits hepatitis B virus replication in mice. Mol. Ther. 8:769-776.

54. Klein, C., et al. 2003. Inhibition of hepatitis B virus replication in vivo by nucleoside analogues and siRNA. Gastroenterology. 125:9-18.

55. Morrissey, D.V., et al. 2005. Activity of stabilized short interfering RNA in a mouse model of hepatitis B virus replication. Hepatology. 41:1349-1356.

56. Sebestyen, M.G., et al. 2006. Mechanism of plasmid delivery by hydrodynamic tail vein injection. I. Hepatocyte uptake of various molecules. J. Gene Med. 8:852-873.

57. Soutschek, J., et al. 2004. Therapeutic silencing of an endogenous gene by systemic administration of modified siRNAs. Nature. 432:173-178.

58. Moore, V., Dunnion, D., Irwin, W.J., and Akhtar, S. 1997. Interactions of hydrophobic oligonucleotide conjugates with the dipeptide transporter in Caco-2 cells. Biochem. Pharmacol. 53:1223-1228.

59. Ebbesen, M., and Jensen, T.G. 2006. Nanomedicine: techniques, potentials, and ethical implications. J. Biomed. Biotechnol. 2006:51516.

60. Li, W., and Szoka, F.C., Jr. 2007. Lipid-based nanoparticles for nucleic acid delivery. Pharm. Res. 24:438-449.

61. Halder, J., et al. 2006. Focal adhesion kinase targeting using in vivo short interfering RNA delivery in neutral liposomes for ovarian carcinoma therapy. Clin. Cancer Res. 12:4916-4924.

62. Sorensen, D.R., Leirdal, M., and Sioud, M. 2003. Gene silencing by systemic delivery of synthetic siRNAs in adult mice. J. Mol. Biol. 327:761-766.

63. Verma, U.N., Surabhi, R.M., Schmaltieg, A., Becerra, C., and Gaynor, R.B. 2003. Small interfering RNAs directed against beta-catenin inhibit the in vitro and in vivo growth of colon cancer cells. Clin. Cancer Res. 9:1291-1300.

64. Arnold, A.S., et al. 2007. Specific beta1-adrenergic receptor silencing with small interfering RNA lowers high blood pressure and improves cardiac function in myocardial ischemia. J. Hypertens. 25:197-205.

65. Khan, A., et al. 2004. Sustained polymeric delivery of gene silencing antisense ODNs, siRNA, DNAzymes and ribozymes: in vitro and in vivo studies. J. Drug Target. 12:393-404.

66. Morrissey, D.V., et al. 2005. Potent and persistent in vivo anti-HBV activity of chemically modified siRNAs. Nat. Biotechnol. 23:1002-1007.

67. Geisbert, T.W., et al. 2006. Postexposure protection of guinea pigs against a lethal ebola virus challenge isconferred by RNA interference. J. Infect. Dis. 193:1650-1657. 
68. Pirollo, K.F., et al. 2007. Materializing the potential of small interfering RNA via a tumor-targeting nanodelivery system. Cancer Res. 67:2938-2943.

69. Chien, P.Y., et al. 2005. Novel cationic cardiolipin analogue-based liposome for efficient DNA and small interfering RNA delivery in vitro and in vivo. Cancer Gene Ther. 12:321-328.

70. Pal, A., et al. 2005. Systemic delivery of RafsiRNA using cationic cardiolipin liposomes silences Raf1 expression and inhibits tumor growth in xenograft model of human prostate cancer. Int. J. Oncol. 26:1087-1091.

71. Semple, S.C., et al. 2005. Immunogenicity and rapid blood clearance of liposomes containing polyethylene glycol-lipid conjugates and nucleic acid. J. Pharmacol. Exp. Ther. 312:1020-1026.

72. Ishida, T., Wang, X., Shimizu, T., Nawata, K., and Kiwada, H. 2007. PEGylated liposomes elicit an anti-PEG IgM response in a T cell-independent manner. J. Control Release. 122:349-355.

73. Kichler, A. 2004. Gene transfer with modified polyethylenimines. J. Gene Med. 6(Suppl. 1):S3-S10.

74. Kircheis, R., Wightman, L., and Wagner, E. 2001. Design and gene delivery activity of modified polyethylenimines. Adv. Drug Deliv. Rev. 53:341-358.

75. Ge, Q., et al. 2004. Inhibition of influenza virus production in virus-infected mice by RNA interference. Proc. Natl. Acad. Sci. U. S. A. 101:8676-8681.

76. Urban-Klein, B., Werth, S., Abuharbeid, S., Czubayko, F., and Aigner, A. 2005. RNAi-mediated gene-targeting through systemic application of polyethylenimine (PEI)-complexed siRNA in vivo. Gene Ther. 12:461-466.

77. Liu, S. 2006. Radiolabeled multimeric cyclic RGD peptides as integrin alphavbeta 3 targeted radiotracers for tumor imaging. Mol. Pharm. 3:472-487.

78. Schiffelers, R.M., et al. 2004. Cancer siRNA therapy by tumor selective delivery with ligand-targeted sterically stabilized nanoparticle. Nucleic Acids Res. 32:e149.

79. Tomalia, D.A., Reyna, L.A., and Svenson, S. 2007. Dendrimers as multi-purpose nanodevices for oncology drug delivery and diagnostic imaging. Biochem. Soc. Trans. 35:61-67.

80. Yoo, H., Sazani, P., and Juliano, R.L. 1999. PAMAM dendrimers as delivery agents for antisense oligonucleotides. Pharm. Res. 16:1799-1804

81. Kang, H., DeLong, R., Fisher, M.H., and Juliano, R.L. 2005. Tat-conjugated PAMAM dendrimers as delivery agents for antisense and siRNA oligonucleotides. Pharm. Res. 22:2099-2106.

82. Tsubouchi, A. 2002. Localized suppression of RhoA activity by Tyr31/118-phosphorylated paxillin in cell adhesion and migration. J. Cell Biol. 159:673-683.

83. Huang, Y.Z., Zang, M., Xiong, W.C., Luo, Z., and Mei, L. 2003. Erbin suppresses the MAP kinase pathway. J. Biol. Chem. 278:1108-1114.

84. Vangasseri, D.P., Han, S.J., and Huang, L. 2005. Lipid-protamine-DNA-mediated antigen delivery. Curr. Drug Deliv. 2:401-406.

85. Song, E., et al. 2005. Antibody mediated in vivo delivery of small interfering RNAs via cell-surface receptors. Nat. Biotechnol. 23:709-717.

86. Takeshita, F., and Ochiya, T. 2006. Therapeutic potential of RNA interference against cancer. Can cer Sci. 97:689-696.

87. Minakuchi, Y., et al. 2004. Atelocollagen-mediated synthetic small interfering RNA delivery for effec- tive gene silencing in vitro and in vivo. Nucleic Acids Res. 32:e109.

88. Hu-Lieskovan, S., Heidel, J.D., Bartlett, D.W., Davis, M.E., and Triche, T.J. 2005. Sequence-specific knockdown of EWS-FLI1 by targeted, nonviral delivery of small interfering RNA inhibits tumor growth in a murine model of metastatic Ewing's sarcoma. Cancer Res. 65:8984-8992.

89. Meade, B.R., and Dowdy, S.F. 2007. Exogenous siRNA delivery using peptide transduction domains/cell penetrating peptides. Adv. Drug Deliv. Rev. 59:134-140.

90. Juliano, R.L. 2006. Intracellular delivery of oligonucleotide conjugates and dendrimer complexes. Ann. N. Y. Acad. Sci. 1082:18-26.

91. Kumar, P., et al. 2007. Transvascular delivery of small interfering RNA to the central nervous system. Nature. 448:39-43.

92. Pardridge, W.M. 2007. shRNA and siRNA delivery to the brain. Adv. Drug Deliv. Rev. 59:141-152.

93. McNamara, J.O., 2nd, et al. 2006. Cell type-specific delivery of siRNAs with aptamer-siRNA chimeras, Nat. Biotechnol. 24:1005-1015.

94. Howard, K.A., et al. 2006. RNA interference in vitro and in vivo using a novel chitosan/siRNA nanoparticle system. Mol. Ther. 14:476-484.

95. Massaro, D., Massaro, G.D., and Clerch, L.B. 2004. Noninvasive delivery of small inhibitory RNA and other reagents to pulmonary alveoli in mice. Am. J. Physiol. Lung Cell Mol. Physiol. 287:L1066-L1070.

96. Medarova, Z., Pham, W., Farrar, C., Petkova, V., and Moore, A. 2007. In vivo imaging of siRNA delivery and silencing in tumors. Nat. Med. 13:372-377.

97. Akhtar, S. 2006. Non-viral vectors for gene therapy: Beyond delivery. Gene Ther. 13:739-740.

98. Kabanov, A.V. 2006. Polymer genomics: an insight into pharmacology and toxicology of nanomedicines. Adv. Drug Deliv. Rev. 58:1597-1621.

99. Tolentino, M.J., et al. 2004. Intravitreal injection of vascular endothelial growth factor small interfering RNA inhibits growth and leakage in a nonhuman primate, laser-induced model of choroidal neovascularization. Retina. 24:132-138.

100.Reich, S.J., et al. 2003. Small interfering RNA (siRNA) targeting VEGF effectively inhibits ocular neovascularization in a mouse model. Mol. Vis. 9:210-216.

101. Nakamura, H., et al. 2004. RNA interference targeting transforming growth factor-beta type II receptor suppresses ocular inflammation and fibrosis. Mol. Vis. 10:703-711.

102.Herard, A.S., et al. 2006. siRNA targeted against amyloid precursor protein impairs synaptic activity in vivo. Neurobiol. Aging. 27:1740-1750.

103.Pille, J.Y., et al. 2005. Anti-RhoA and anti-RhoC siRNAs inhibit the proliferation and invasiveness of MDA-MB-231 breast cancer cells in vitro and in vivo. Mol. Ther. 11:267-274.

104.Kim, W.J., et al. 2006. Cholesteryl oligoarginine delivering vascular endothelial growth factor siRNA effectively inhibits tumor growth in colon adenocarcinoma. Mol. Ther. 14:343-350.

105.Li, S., et al. 2007. Polyethylenimine-complexed plasmid particles targeting focal adhesion kinase function as melanoma tumor therapeutics. Mol. Ther. 15:515-523.

106.Kishida, T., et al. 2004. Sequence-specific gene silencing in murine muscle induced by electropora- tion-mediated transfer of short interfering RNA. J. Gene Med. 6:105-110.

107. Golzio, M., Mazzolini, L., Moller, P., Rols, M.P., and Teissie, J. 2005. Inhibition of gene expression in mice muscle by in vivo electrically mediated siRNA delivery. Gene Ther. 12:246-251.

108.Akaneya, Y., Jiang, B., and Tsumoto, T. 2005. RNAiinduced gene silencing by local electroporation in targeting brain region. J. Neurophysiol. 93:594-602.

109.Dorn, G., et al. 2004. siRNA relieves chronic neuropathic pain. Nucleic Acids Res. 32:e49.

110.Tan, P.H., Yang, L.C., Shih, H.C., Lan, K.C., and Cheng, J.T. 2005. Gene knockdown with intrathecal siRNA of NMDA receptor NR2B subunit reduces formalin-induced nociception in the rat. Gene Ther. 12:59-66.

111.Makimura, H., Mizuno, T.M., Mastaitis, J.W., Agami, R., and Mobbs, C.V. 2002. Reducing hypothalamic AGRP by RNA interference increases metabolic rate and decreases body weight without influencing food intake. BMC Neurosci. 3:18.

112.Shishkina, G.T., Kalinina, T.S., and Dygalo, N.N. 2004. Attenuation of alpha2A-adrenergic receptor expression in neonatal rat brain by RNA interference or antisense oligonucleotide reduced anxiety in adulthood. Neuroscience. 129:521-528.

113.Thakker, D.R., et al. 2004. Neurochemical and behavioral consequences of widespread gene knockdown in the adult mouse brain by using nonviral RNA interference. Proc. Natl. Acad. Sci. U. S. A. 101:17270-17275.

114.Zhang, X., et al. 2004. Small interfering RNA targeting heme oxygenase- 1 enhances ischemiareperfusion-induced lung apoptosis. J. Biol. Chem. 279:10677-10684.

115.Bitko, V., Musiyenko, A., Shulyayeva, O., and Barik, S. 2005. Inhibition of respiratory viruses by nasally administered siRNA. Nat. Med. 11:50-55.

116.Song, E., et al. 2003. RNA interference targeting Fas protects mice from fulminant hepatitis. Nat. Med. 9:347-351.

117. Thomas, M., Lu, J.J., Chen, J., and Klibanov, A.M. 2007. Non-viral siRNA delivery to the lung. $A d v$. Drug Deliv. Rev. 59:124-133.

118.Li, B.J., et al. 2005. Using siRNA in prophylactic and therapeutic regimens against SARS coronavirus in Rhesus macaque. Nat. Med. 11:944-951.

119.Filleur, S., et al. 2003. SiRNA-mediated inhibition of vascular endothelial growth factor severely limits tumor resistance to antiangiogenic thrombospondin- 1 and slows tumor vascularization and growth. Cancer Res. 63:3919-3922.

120.Palliser, D., et al. 2006. An siRNA-based microbicide protects mice from lethal herpes simplex virus 2 infection. Nature. 439:89-94.

121. Hassani, Z., et al. 2005. Lipid-mediated siRNA delivery down-regulates exogenous gene expression in the mouse brain at picomolar levels. J. Gene Med. 7:198-207.

122.Kim, W.J., Chang, C.W., Lee, M., Kim, S.W. 2007. Efficient siRNA delivery using water soluble lipopolymer for anti-angiogenic gene therapy. J. Control Release. 118:357-363.

123.Peer, D., Zhu, P., Carman, C.V., Lieberman, J., and Shimaoka, M. 2007. Selective gene silencing in activated leukocytes by targeting siRNAs to the integrin lymphocyte function-associated antigen-1. Proc. Natl. Acad. Sci. U. S. A. 104:4095-4100. 